\title{
Is standing sway an accurate measure of fall risk and predictor of future falls in older adults?
}

\section{TIBOR HORTOBÁGYI ${ }^{1}$ | LUIS A. TEIXEIRA ${ }^{2}$ | JACQUES DUYSENS ${ }^{3}$ | URS GRANACHER ${ }^{4}$ | JAAP VAN DIEËN ${ }^{5}$ | RENATO MORAES 6}

\footnotetext{
${ }^{1}$ Center for Human Movement Sciences, University Medical Center Groningen, The University of Groningen, Groningen, The Netherlands

2 Human Motor Systems Laboratory, School of Physical Education and Sport, University of São Paulo, São Paulo, Brazil

${ }^{3}$ Motor Control Laboratory, Movement Control and Neuroplasticity Research Group KU Leuven, Leuven, Belgium

4 Division of Training and Movement Sciences, Research Focus Cognition Sciences, University of Potsdam, Potsdam, Germany

${ }^{5}$ Department of Human Movement Sciences, Faculty of Behavioural and Movement Sciences, Vrije Universiteit Amsterdam, The Netherlands

${ }^{6}$ Biomechanics and Motor Control Laboratory, School of Physical Education and Sport of Ribeirão Preto, University of São Paulo, São Paulo, Brazil
}

Correspondence to: Tibor Hortobágyi, Center for Human Movement Sciences, University Medical Center Groningen, The University of Groningen, Groningen, The Netherlands.

email: t.hortobagyi@umcg.nl

https://doi.org/10.20338/bjmb.v14i01.176

PUBLICATION DATA

Received 28032020

Accepted 30032020

Published 01042020

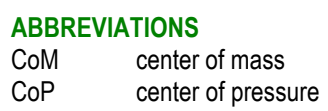

In 1836, English physician Marshall Hall in his lectures on the nervous system posited that proprioception, vestibular function, and vision underlie postural control. In 1851, German physician Moritz Heinrich Romberg observed that patients suffering from tabes dorsalis lost their balance when they stood with eyes closed, giving rise to the Romberg test. In the 1880s, American neurologist Silas Weir Mitchell developed the first standing sway meter. This rudimentary device and the clinical observations over 180 years ago formed the neuromechanical basis of today's postural sway measurements during quiet standing tasks on a force platform used to 'measure' fall risk and 'predict' future falls.

Annually, $30-50 \%$ of adults age $65-90$ experience a fall. Falls are the leading cause of fatal and non-fatal injuries and will result in 3M injuries, 27,000 deaths, and incur medical and social costs of $\sim$ \$67B in 2020 in the USA. In Brazil and in many other countries, including the Netherlands, public healthcare reports reveal a trend toward increasing rates of fallrelated morbidity and mortality among older adults. The most frequently declared causes of falls among the elderly being tripping, slipping, dizziness, and uneven flooring ${ }^{1}$. A massive international research effort is underway to identify accurate measures of fall risks and predict future falls. Such data could inform therapists to develop targeted interventions to reduce fall risks and delay a first-ever fall in older adults.

While the measurement of postural sway is relatively straightforward, it is challenging to establish a mechanistic link between the magnitude and velocity of postural sway and fall risk as well as future falls. Fundamental inconsistencies between studies complicate our current understanding and perhaps even the validity of postural sway during balancing tasks as a predictor of fall risk and future falls. In standing, muscles generate corrective moments to counteract gravity's pull but a putative role has been also assigned to the availability and accuracy of sensory information, sensory weighting, delays and gains of control loops, and system noise. Muscle moments shift the center of pressure (CoP) and 
Brazilian Journal of Motor Behavior

control center of mass (CoM) movements, which, if 'too large' or 'variable', are presumably undesirable and should be limited by feedforward or feedback mechanisms. Like Romberg's ill patients with a lesioned dorsal column, healthy older adults also increase sway magnitude in standing with eyes closed. Nevertheless, older adults with a history of falls are able to modulate and reduce their postural sway when performing a visual-cognitive task (the adapted visual Stroop test) ${ }^{2}$. Heightening the conflicting data further are the observations that Parkinsonians often show less sway than healthy controls but are more fall prone. Then, there is no clear understanding of the source and meaning of postural sway. Some authors posit that sway is not even a valid measure of postural control with respect to fall prediction because metabolic costs increase with decreasing sway. From this perspective, the variable neuromuscular system optimizes in standing would not be CoP or CoM sway magnitude and variability but metabolic cost. It is also not possible to make inferences on the functionality of postural responses to perturbations because we cannot tell if the motor actions observed accelerate the CoM towards or away from the target state ${ }^{3}$. There is, then, the view assigning a beneficial, 'exploratory' role to a 'certain magnitude' of sway4. Thus, we cannot tell how much of sway is 'good' or 'bad' for estimating balance stability or its association with fall prevention. Our opinion resonates with the conclusion that the fall risk assessment tools, including standing sway CoP metrics, currently in use to test older adults, do not have sufficiently high predictive validity for differentiating high and low fall risks and to predict future falls on an individual level ${ }^{5}$. Falls also often occur in dynamic situations and rarely during quiet standing.

The future perspective is that age-related increase in balance sway magnitude could be a valid risk factor for falls and predict future falls if we could verify that sway magnitude or velocity in unperturbed standing were markers of neural and mechanical dysfunctions that also fail at the time of a fall. That is, we need to link sway outcomes, measured during standing under sensory challenges, to dysfunctions at the time of a fall. Until we have such data, it remains indeed difficult to explain why standing trials with eyes opened instead of eyes closed predicted future falls more accurately in 1,877 community-dwelling adults age $70^{6}$ and why inexplicably both anterior-posterior and mediolateral CoP velocity predicted future falls $\mathbf{s}^{7,8}$. We need to develop innovative experiments to understand the relationship between sensory acuity, sensory processing, sensory and motor noise, contractile properties of key muscles and standing sway and see if changes in these outcomes would reduce fall risks and prevent future falls ${ }^{9}$. There is also a need to complement the static CoP measures with dynamic ones, provided by moving platforms ${ }^{10}$.

\section{REFERENCES}

1. Leitão SM, Oliveira SC, Rolim LR, Carvalho Filho RP. Epidemiology of falls in older adults in Brazil: an integrative literature review. Geriatr Gerontol Aging 2018;12:172-9.

2. Batistela RA, Oates $A$, Moraes R. Haptic information and cognitive-visual task reduce postural sway in faller and non-faller older adults. Hum Mov Sci 2018;60:150-61.

3. Houdijk H, Brown SE, van Dieen JH. Relation between postural sway magnitude and metabolic energy cost during upright standing on a compliant surface. J Appl Physiol (1985) 2015;119(6):696-703. 
Brazilian Journal of Motor Behavior

\section{Current Opinion}

4. van Emmerik RE, van Wegen $\mathrm{EE}$. On the functional aspects of variability in postural control. Exerc Sport Sci Rev 2002;30(4):177-83.

5. Park SH. Tools for assessing fall risk in the elderly: a systematic review and meta-analysis. Aging Clin Exp Res 2018;30(1):1-16.

6. Johansson J, Nordstrom A, Gustafson Y, Westling G, Nordstrom P. Increased postural sway during quiet stance as a risk factor for prospective falls in community-dwelling elderly individuals. Age Ageing 2017;46(6):964-70.

7. Maki BE, Holliday PJ, Topper AK. A prospective study of postural balance and risk of falling in an ambulatory and independent elderly population. J Gerontol 1994;49(2):M7284.

8. Kwok BC, Clark RA, Pua YH. Novel use of the Wii Balance Board to prospectively predict falls in community-dwelling older adults. Clin Biomech (Bristol, Avon) 2015;30(5):481-4.

9. Barbosa RN, Silva NRS, Santos DPR, Moraes R, Gomes MM. Force stability training decreased force variability of plantar flexor muscles without reducing postural sway in female older adults. Gait Posture 2020;77:288-92.

10. Ribeiro de Souza C, Betelli MT, Takazono PS, de Oliveira JA, Coelho DB, Duysens J et al. Evaluation of balance recovery stability from unpredictable perturbations through the compensatory arm and leg movements (CALM) scale. PLoS One 2019;14(8):e0221398.

Citation: Hortobágyi T, Teixeira LA, Duysens J, Granacher U, Van Dieën J, Moraes R. Is standing sway an accurate measure of fall risk and predictor of future falls in older adults?. BJMB. 2020: 14(1): 1-3.

Editors: Dr Fabio Augusto Barbieri - São Paulo State University (UNESP), Bauru, SP, Brazil; Dr José Angelo Barela São Paulo State University (UNESP), Rio Claro, SP, Brazil; Dr Natalia Madalena Rinaldi - Federal University of Espírito Santo (UFES), Vitória, ES, Brazil.

Section Editors (Current Opinion): Dr Luis Augusto Teixeira - University of São Paulo (USP), São Paulo, SP, Brazil; Dr Tibor Hortobágyi - University of Groningen, The Netherlands; Dr Renato de Moraes - University of São Paulo (USP), Ribeirão Preto, SP, Brazil.

Copyright:@ 2020 Hortobágyi, Teixeira, Duysens, Granacher, Van Dieën and Moraes and BJMB. This is an openaccess article distributed under the terms of the Creative Commons Attribution-Non Commercial-No Derivatives 4.0 International License which permits unrestricted use, distribution, and reproduction in any medium, provided the original author and source are credited.

Funding: There was no funding for this study.

Competing interests: The authors have declared that no competing interests exist.

DOI: https://doi.org/ 10.20338/bjmb.v14i01.176 